ARTICLE

\title{
Micro-Scale Dose Distribution of Microplanar X Rays from Synchrotron Radiation: Measurement and Monte Carlo Calculation
}

\author{
Nobuteru NARIYAMA ${ }^{1, *}$, Keiji UMETANI ${ }^{1}$, Kunio SHINOHARA ${ }^{2}$, \\ Takeshi KONDOH $^{3}$, Ai KURIHARA ${ }^{4}$ and Manabu FUKUMOTO ${ }^{4}$ \\ ${ }^{1}$ Japan Synchrotron Radiation Research Institute, Kouto 1-1-1, Sayo, Hyogo 679-5198, Japan \\ ${ }^{2}$ Waseda University, Advanced Research Institute for Science and Engineering, Shinjuku, Tokyo 169-8555, Japan \\ ${ }^{3}$ Kobe University, Graduate School of Medicine, Kobe, Hyogo 650-0017, Japan \\ ${ }^{4}$ Tohoku University, Institute of Development, Aging and Cancer, Sendai, Miyagi 980-8575, Japan
}

\begin{abstract}
Knowledge of dose distribution with micron resolution is required in radiation therapy studies that use a spatially fractionated X-ray microbeam. However, the condition of the collimator affects the dose distribution. To estimate the performance of a modified collimator, the measured dose distribution was compared with that of a Monte Carlo PENELOPE calculation. The dose distribution for synchrotron radiation with an effective energy of $90 \mathrm{keV}$ that passed through a single-slit collimator to produce a $25-\mu \mathrm{m}$-wide beam was measured with a GafChromic HD-810 film and digital microscope. The calculated and measured doses did not agree in the slope because the measured dose distribution was partly enhanced by photons that were reflected from the collimator. In the other region, the doses agreed within $20 \%$. Compared with a former multi-slit collimator, the single-slit collimator considerably reduced the scattered components and produced a lower dose in the valley, which approached the calculated dose.
\end{abstract}

KEYWORDS: microbeam, synchrotron radiation, PENELOPE, GafChromic film

\section{Introduction}

Most general-purpose photon Monte Carlo (MC) transport codes incorporate electron transport. For a photon source, however, the kerma approximation is enough in most cases because the traveling path of electrons is much shorter than that of photons. The consideration of electron transport is necessary in determining the dose distribution in a non-electric equilibrium because the transient region is too thin in a solid except in the case of high-energy photons; for example, only $140 \mu \mathrm{m}$ in water at $100 \mathrm{keV}$. In other words, the electron transport is important for the dose distribution with micro-scale resolution.

Recently, spatially fractionated radiation therapy employing GRID therapy has been used in medical treatment to debulk tumors with an MV photon beam. ${ }^{1,2)}$ The opening separation is several centimeters. Normal tissue is expected to be better able to recover in the treatment, in which the space-dependent dose delivery plays an important role.

As another type of spatially fractionated radiation therapy, the use of a multi-slit microbeam of synchrotron radiation has been reported to enhance the tolerance of normal tissue to radiation, ${ }^{3-6)}$ and microbeam radiation therapy (MRT) has recently been investigated at the synchrotron radiation facilities SPring- $8^{7)}$ and ESRF. The typical slit width was $25 \mu \mathrm{m}$ and the center-to-center separation was $200 \mu \mathrm{m}$. Grid irradiation outperformed parallel-slit irradiation in the treatment of rats. The remedial value is considered to depend on the peak-to-valley dose ratio, and

*Corresponding author, E-mail: nariyama@ spring8.or.jp

(C) 2011 Atomic Energy Society of Japan, All Rights Reserved. thus, there have been extensive measurements and $\mathrm{MC}$ calculations. Because effective X-ray beam energies are about $100 \mathrm{keV}$, the dose in the valley is not negligible; i.e., electron transport needs to be considered even for the photon source in the MC calculation.

A dose distribution in too thin region makes the measurement difficult. The requirements for the dosimeter are micron resolution, tissue equivalence and a wide dose range. Micron resolution is considered to be attained using thin material or highly homogeneous material with a two-dimensional reader. While the dose range can be compensated somewhat by changing the irradiation time for the dosimeter, sensitivity is improved at the expense of resolution. Tissue equivalence is indispensable in the low-energy region because the response depends largely on the photon energy.

We applied LiF thermoluminescent (TL) dosimeters and a TL shee ${ }^{8)}$ to the microbeam, and observed the TL with a microscope and charge-coupled device (CCD) camera. However, the particle size of LiF elements was large and an image with the required resolution could not be obtained. GafChromic film was a promising candidate because its micron imaging resolution and wide dose range are well known. For application to the present problem, the reliability of the dose estimation needs to be confirmed on a micron scale.

We tested three kinds of GafChromic films HD-810, MD-55 and EBT, and found that only HD-810, which is the thinnest of the three, had a reasonable dose with satisfactory resolution for the multi-slit beam. For the imaging reader, a film scanner and a flat-bed scanner having a resolution of 


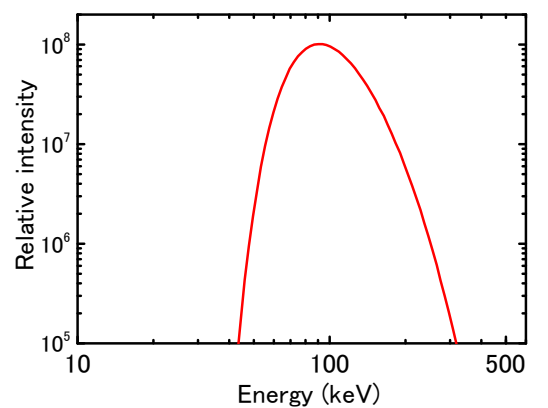

Fig. 1 Source photon fluence through a 3-mm-thick copper sheet at the BL28B2 beamline calculated with the SPECTRA $\operatorname{code}^{12)}$

5400 dpi were tested; in spite of the high resolution of the sensor, the focusing was not so sharp and the multi-slit lines were not clearly imaged. As a result, only the combination of an optical microscope ${ }^{9-11)}$ and a CCD camera provided satisfactory focusing. A lens with $20 \times$ magnification gave the finest resolution; at 50× magnification, polymer particles appeared, which was not desired for the dose measurement. Because the focusing can be done manually and visually confirmed immediately, the reproducibility does not depend on the operator; this is the advantage of the conventional microscope.

To enhance the accuracy, we used band-pass filters to achieve linearity between the dose and optical density (OD). By confirming the linearity, the reading condition can be checked. Instead of increased accuracy, however, the dose range was narrower. To widen the range, we used two absorption peaks around 610 and $670 \mathrm{~nm}$, which provided different sensitivities: a high dose at $610 \mathrm{~nm}$ and low dose at $670 \mathrm{~nm}$.

At SPring-8, the micro-slit beam was obtained through a multi-slit collimator, a stack of Kapton and tungsten. We have measured the dose distribution but the valley dose was larger by a factor of 2.3 than MC calculations. The collimator was replaced with a variable single-slit collimator. For multi-slit irradiation, multiple shots of the single slit beam are used. The advantages are the changeable slit width and center-to-center distance, and the high resistance to radiation damage. In this study, the dose distribution was measured for microbeam $\mathrm{X}$ rays passing through the single-slit collimator by digital microscopy with GafChromic film, and the results were compared with $\mathrm{MC}$ calculations obtained using $\mathrm{MC}$ code to examine the performance of the collimator.

\section{Materials and Methods}

\section{Irradiation Condition}

The experiment was performed at the bending-magnet beamline BL28B2 of SPring-8. The ring current was maintained at approximately $100 \mathrm{~mA}$ in top-up operation. White $\mathrm{X}$ rays were filtered with a 3-mm-thick copper sheet, resulting in $90 \mathrm{keV}$ peak energy. Figure 1 shows the energy distribution calculated with the SPECTRA code. ${ }^{12)}$ The air kerma rate was measured using a free-air ionization chamber for high-energy photons and determined to be $150 \mathrm{~Gy} / \mathrm{s}^{13}$ )
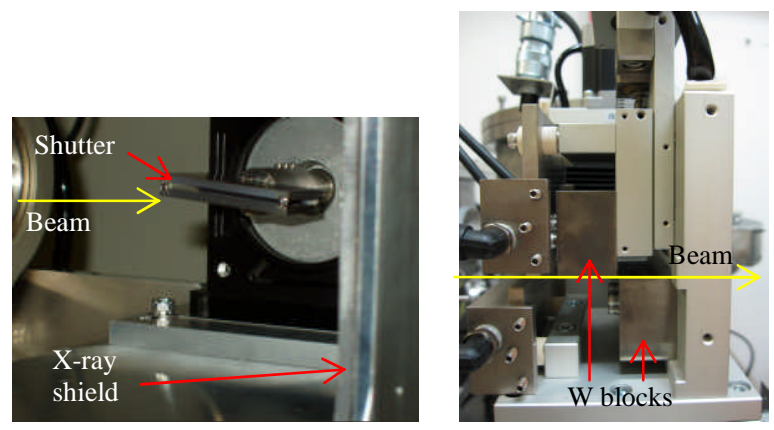

Fig. 2 Photographs of beamline components: fast tantalum shutter (left) and variable-gap single-slit collimator (right). The collimator is formed by 2 -cm-thick tungsten blocks placed above and below the beam. To avoid scattering of $\mathrm{X}$ rays from these components, an X-ray shield was positioned as shown in the photo on the left.

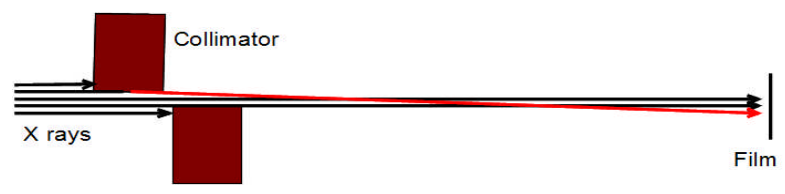

Fig. 3 Total reflection (red line) occurs at the upper block after the lower block is adjusted when the surface of the collimator is not completely parallel to the beam.

An inhomogeneous intensity distribution was observed in the vertical direction.

For the bending-magnet beamline, the emittance is $64 \mu \mathrm{rad}$ in the vertical direction, almost parallel to the beam, and the beam can be clearly cut into microbeams. This is a remarkable advantage over using conventional $\mathrm{X}$ rays. Figure 2 shows side views of the fast rotary-solenoid-driven tantalum shutter and the single-slit collimator. The irradiation time was determined with the shutter, which was positioned before the collimator at an angle to the beam. To allow the beam to pass through the tantalum shutter must only rotate slightly enabling shutter times as fast as $0.1 \mathrm{~s}$. To form the collimator, two tungsten blocks $2 \mathrm{~cm}$ in thickness and $3 \mathrm{~cm}$ in height were set above and below the beam. Their position and orientation were controlled with $\mathrm{X}, \mathrm{Y}$ and $\mathrm{Z}$ stages and a goniometer. The resolution of the slit width was $0.5 \mu \mathrm{m}$.

When the collimator is tilted even slightly, scattering and total reflection occur as shown in Fig. 3, and a dark broad line appears on the film accompanying the slit beam. Total reflection is observed only when the collimator surface is almost flat and the photons are incident on it at an angle smaller than the critical angle. The critical angle $\theta_{c}(\mathrm{mrad})$ is expressed as

$$
\theta_{C}=20 \rho^{1 / 2} / E
$$

where $\rho\left(\mathrm{g} / \mathrm{cm}^{3}\right)$ is the material density and $E(\mathrm{keV})$ is the photon energy. ${ }^{14)}$ For tungsten, the critical angle is $1.46 \mathrm{mrad}$ at $60 \mathrm{keV}$, that is, $0.08^{\circ}$, which is larger than the beam emittance. In the experiment, the collimator was set to reduce total reflection by adjusting the lower tungsten block. 

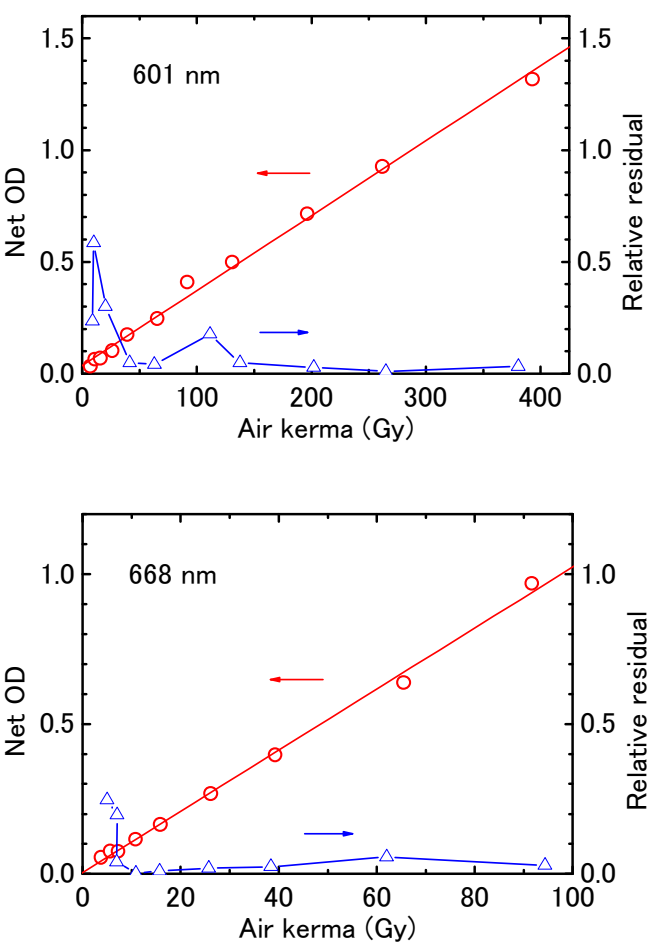

Fig. 4 Calibration curves for the HD-810 film: net OD versus air kerma data (red circle) at 601-nm (top panel) and 668-nm (bottom panel) light. The red line was obtained by a least-squares fit to the data. The blue triangles denote the relative residuals of the regression line.

Because the upper block could not be moved independently, total reflection from the upper block inevitably appeared.

GafChromic HD-810 film has an almost bare radiation-sensitive layer on its polyester side. ${ }^{14)}$ The reverse side of the film was irradiated with $\mathrm{X}$ rays for 1 and $10 \mathrm{~s}$ for the peak and valley regions, respectively.

\section{Microscopic Dose Measuring Method}

The microscopic dosimetry system has the following components: an optical microscope with a $20 \times$ lens, changeable bandpass filters, an X-Y motorized stepper stage, a 12-bit gray-scale CCD camera with a pixel size of $6.45 \mu \mathrm{m}$, a personal computer and imaging analysis software. ${ }^{11)}$ The image is focused manually for each shot. The field of view at one shot was nearly $0.4 \mathrm{~mm}$ square. Because the surrounding light influences the readout, the entire system is placed in a dark room.

The light intensity of the irradiated HD-810 film was converted into OD. Then, the OD value was converted to a dose in the film with the calibration curves shown in Fig. 4; the air kerma was converted to the film dose. The calibration was performed at the same beamline where the microbeam was supplied, thereby reducing the uncertainties caused by the energy response. The addition of extra metal filters in the beam path decreased the beam intensity, and by varying the beam intensity and the irradiation time, the calibration curve was obtained.

The experimental uncertainties mainly originated from the

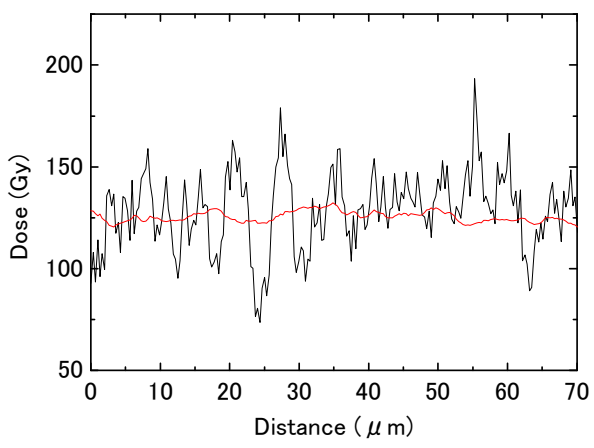

Fig. 5 Dose distribution for $100-\mu \mathrm{m}$-wide horizontal slit beam: data taken on a line that is one pixel wide (black line) and then horizontally averaged (red line).

film's calibration, dose rate effect and uniformity. The uncertainty from the calibration was $6 \%$ above $6 \mathrm{~Gy}$, but increased to $25 \%$ at $3.75 \mathrm{~Gy}$ for $668 \mathrm{~nm}$ light, as shown in Fig. 4. A dose rate effect of $5 \%{ }^{15)}$ was included in the above uncertainties. Figure 5 shows the measured dose distributions in the case of a broad $100-\mu \mathrm{m}$-wide slit beam for data taken from a line that is one pixel wide (black line) and then horizontally averaged (red line). The reading along the one-pixel-wide line had a large scatter with an uncertainty of $15 \%$, but the averaged values were constant with an uncertainty of $2 \%$.

\section{Monte Carlo Calculation}

The PENELOPE2008 code ${ }^{16)}$ was used for the Monte Carlo (MC) calculation. The code adopted a "mixed simulation", in which a hard collision is simulated as a single collision and a soft collision is treated as multiple scattering. In this code, SBOX and SCONE functions were used to define source conditions such as the beam area and beam direction. This code considers polarization but does not consider the anisotropic emission of photoelectrons and Compton electrons.

In the calculations, a rectangular beam with a height of $25 \mu \mathrm{m}$ and a width of $20 \mathrm{~mm}$ was injected to $100-\mu \mathrm{m}$-thick polyester, as shown in Fig. 6, and the doses in the polyester were calculated. The scoring bin in the vertical direction was set to $3.1 \mu \mathrm{m}$. The output doses were converted to those for the photon fluence at which an air kerma of $150 \mathrm{~Gy}$ is obtained for a broad beam by using the spectrum in Fig. 1. The computer that was used had an Intel Core2 Duo processor $(\mathrm{E} 84003.0 \mathrm{GHz})$ and a Windows operating system, and the compiler was Lahey Fortran 95 ver. 5.5.

\section{Results and Discussion}

\section{Comparison between Experiment and MC Calculation}

The measured and calculated dose distributions for the $25-\mu$ m-wide slit beam are shown in Fig. 7. The experimental result was converted from one of the images obtained from nearly a hundred films irradiated. While in the beam all the results mostly agreed within the error bars, in the slope the measured distribution more or less changed depending on the collimator and film conditions. The result of Fig. 7 was 


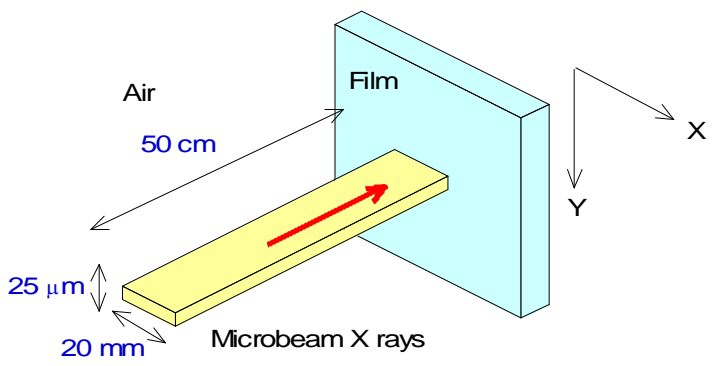

Fig. 6 Geometry for MC calculations. A $20 \mathrm{~mm} \times 25 \mu \mathrm{m}$ rectangular beam was incident on the film through a 50 -cm-thick air layer. Dose distribution in the vertical direction, which was defined as Y-axis direction, was calculated.

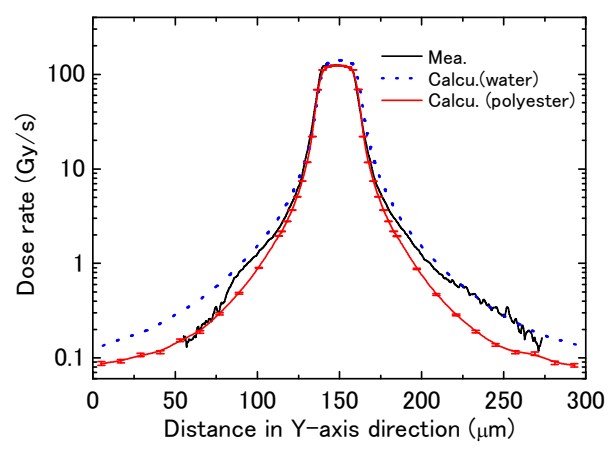

Fig. 7 Measured dose distribution in the film (black) compared with MC calculated dose distributions in water (blue) and polyester (red) for a $25-\mu \mathrm{m}$ slit beam. In the calculation, the source intensity in Fig. 1 is considered. The error bars express $1 \sigma$ uncertainties.

considered to be obtained at the relatively simple condition. The distinguishing feature was that the measured distribution was asymmetric. The dose for the right valley, i.e., the lower valley was enhanced presumably by total-reflection photons from the upper block. It is possible that the two surfaces were not completely parallel, and the upper tungsten block reflected the photons. The error bars on the MC calculated doses express $1 \sigma$ statistical uncertainties; these uncertainties were below $0.1 \%$ in the beam and below $4.2 \%$ in the valley.

Figure 8 shows the ratio of the calculated dose values to the experimental values. Near the edge of the beam, disagreement that corresponded to an unknown streak image on the film was seen. On the left, i.e., the upper valley, an unnatural increase in the measured dose occurred between 77 and $113 \mu \mathrm{m}$, possibly also induced by reflection photons from the surface of the collimator. This dark banding on the film was not observed when the slit size was changed to $100 \mu \mathrm{m}$. In the other region, the measured and calculated values agreed within $20 \%$.

The measured GafChromic film dose in the electric equilibrium region can be converted to that in water. In the non-equilibrium region of the valley, however, an accurate conversion is difficult because the electron stopping power, density and photon cross-section differ.

The calculated dose distribution in water is shown in Fig. 7. Compared to the calculated dose distribution in poly-

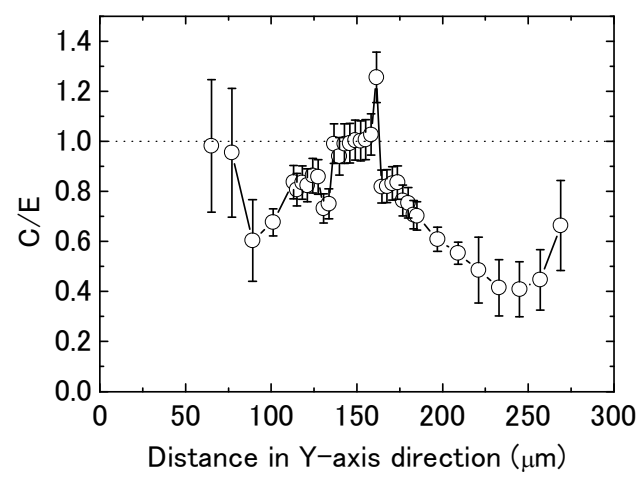

Fig. 8 Ratios of the calculated doses in polyester to the experimental doses shown in Fig. 7

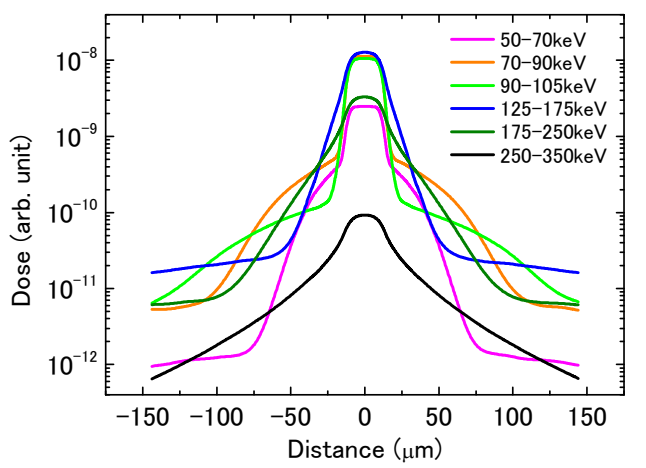

Fig. 9 Contribution to dose distribution in water for incident $X$ rays of various energies in a $25-\mu \mathrm{m}$-wide slit beam. The source intensity in Fig. 1 was considered. The $70-90 \mathrm{keV}$ photons dominate below $70 \mu \mathrm{m}$, and the $90-105 \mathrm{keV}$ photons dominate above $70 \mu \mathrm{m}$.

ester, that in water was $13 \%$ higher in the beam and two times higher in the valley. Because the densities of water and polyester are 1.0 and $1.4 \mathrm{~g} / \mathrm{cm}^{3}$, respectively, the electron range increased, resulting in the dose increase in the valley.

\section{Energy Dependence of Valley Dose}

As shown in Fig. 1, the photon energy of $X$ rays in the beamline predominantly ranged between 40 and $300 \mathrm{keV}$, peaking at $90 \mathrm{keV}$. To demonstrate the contribution of each energy component to the valley dose, the dose distribution in water for monoenergetic $\mathrm{X}$ rays was calculated for the same geometry as shown in Fig. 6. The results are presented in Fig. 9, in which the dose per incident photon is multiplied by the source intensity in Fig. 1 and the energy bin. This figure shows that the shoulder is produced by the $70-90 \mathrm{keV}$ photons with the 90-105 keV photons more influential at a longer distance from the beam.

The calculations presented in Figs. 7 and 9 assumed an ideal condition at the collimator; no photons are incident on the collimator surface, and the valley dose is given by Compton electrons and photoelectrons from the beam. In Fig. 9, the distribution at $125-175 \mathrm{keV}$ is the narrowest because although the Compton electrons are dominant, the energies of approximately $40 \mathrm{keV}$ are too low. 


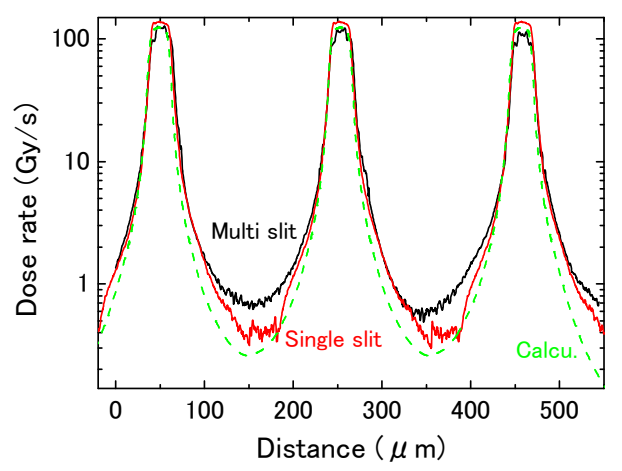

Fig. 10 Dose distributions experimentally obtained when using single-slit and multi-slit ${ }^{11)}$ collimators and those obtained assuming no scattering and reflection at the collimator by $\mathrm{MC}$ calculation. The width of each slit was $25 \mu \mathrm{m}$ and the center-to-center distance of the multi-slit collimators was $200 \mu \mathrm{m}$. The single-slit beam required three shots of $\mathrm{X}$ rays. The calculated dose distribution is the same as that in Fig. 7.

The photon spectra did not differ whether air or vacuum existed before the water; i.e., the scattering of photons by air was not influential. The gradient observed in Figs. 7 and 9 almost agreed with the range of photoelectrons and Compton electrons. That is, the dose in the valley corresponded to the electrons emitted from the X-ray beam for the ideal condition of no photons incident on the collimator surface.

\section{Multi-Slit vs. Single-Slit Beam}

A multi-slit collimator was used in a previous experiment. ${ }^{11)}$ The collimator was formed by alternatively stacking $175-\mu \mathrm{m}$-thick tungsten foils and $25-\mu \mathrm{m}$-thick Kapton films. Figure 10 compares the dose distributions for the single-slit and multi-slit beams. The distribution of the single-slit beam was obtained by introducing a $200-\mu \mathrm{m}$ separation in the distribution of Fig. 7. The tungsten foils in the multi-slit collimator appeared not to be completely parallel to each other. Thus, using the single-slit collimator decreased the valley dose and allowed better adjustment of the orientation; the valley dose closely approached the calculated dose in the case of the single-slit collimator.

\section{Conclusion}

The micro-scale dose distribution for a microbeam from a single-slit collimator was measured with a digital microscopic film system. In the region not affected by the reflected components, the agreement of the MC calculation and the experimental data was within $20 \%$. The MC calculation contributed to the understanding of the phenomenon at the collimator. For the ideal condition, each block of the collimator must be moved independently, thereby enabling the collimator surfaces to be completely parallel to the beam, even if the beam diverges slightly.

If the film records a dense chromatic image, a similar gradient of shade appears adjacent to the beam corresponding to the film resolution. In this case, a symmetric distribution in both valleys would be imaged.

\section{Acknowledgment}

This work was partially supported by Grant-in-Aid for Scientific Research (B). The synchrotron-radiation experiments were performed at the BL28B2 in the SPring- 8 with the approval of the Japan Synchrotron Radiation Research Institute (JASRI) (Proposal No. 2008A1206, 2008B1083, 2009A1158).

\section{References}

1) M. Mohiuddin, M. Fujita, W. F. Regine, A. S. Megooni, G. S. Ibbott, M. M. Ahmed, "High-dose spatially-fractionated radiation (GRID): new paradigm in the management of advanced cancers," Int. J. Radiat. Oncol. Biol. Phys., 45, 721-727 (1999).

2) J. A. Peñagarícano, E. G. Moros, V. Ratanatharathorn, Y. Yan, P. Corry, "Evaluation of spatially fractionated radiotherapy (GRID) and definitive chemoradiotherapy," Int. J. Radiat. Oncol. Biol. Phys., 76, 1369-1375 (2010).

3) D. N. Slatkin, P. Spanne, F. A. Dilmanian, M. Sandborg, "Microbeam radiation therapy," Med. Phys., 19, 1395-1400 (1992).

4) D. N. Slatkin, P. Spanne, F. A. Dilmanian, J.-O. Gebbers, J. A. Laissue, "Subacute neuropathological effects of microplanar beams of x-rays from a synchrotron wiggler," Proc. Natl. Acad. Sci. USA, 92, 8783-8787 (1995).

5) J. A. Laissue, G. Geiser, P. O. Spanne, F. A. Dilmanian, J.-O. Gebbers, M. Geiser, X.-Y. Wu, M. Makar, P. L. Micca, M. M. Nawrocky, D. D. Joel, D. N. Slatkin, "Neuropathology of ablation of rat gliosarcomas and contiguous brain tissues using a microplanar beam of synchrotron-wiggler-generated $\mathrm{x}$ rays," Int. J. Cancer, 78, 654-660 (1998).

6) F. A. Dilmanian, T. M. Button, G. Le Duc, N. Zhong, L. A. Peña, J. A. L. Smith, S. R. Martinez, T. Bacarian, J. Tammam, B. Ren, P. M. Farmer, J. Kalef-Ezra, P. L. Micca, M. M. Nawrocky, J. A. Niederer, F. P. Recksies, A. Fuchs, E. M. Rosen, "Response of rat intracranial 9L gliosarcoma to microbeam radiation therapy," Neuro Oncol., 4, 26-38 (2002).

7) G. Kashino, T. Kondoh, N. Nariyama, K. Umetani, T. Ohigashi, K. Shinohara, A. Kurihara, M. Fukumoto, H. Tanaka, A. Maruhashi, M. Suzuki, Y. Kinashi, Y. Liu, S. Masunaga, M. Watanabe, K. Ono, "Induction of DNA double-strand breaks and cellular migration through bystander effects in cells irradiated with the slit-type microplanar beam of the SPring-8 synchrotron," Int. J. Radiat. Oncol. Biol. Phys., 74, 229-236 (2009).

8) N. Nariyama, A. Konnai, S. Ohnishi, A. Yamaji, N. Ozasa, Y. Ishikawa, "Tissue-equivalent TL sheet dosimetry system for $\mathrm{X}$ - and gamma-ray dose mapping," Radiat. Prot. Dosim., 120, 136-139 (2006).

9) K. Y. Lee, K. K. L. Fung, C.S. Kwok, "Development and initial evaluation of a spectral microdensitometer for analysing radiochromic films," Phys. Md. Biol., 49, 5171-5183 (2004).

10) B. M. Keller, C. Peressotti, J. Pignol, "Optical imaging analysis of microscopic radiation dose gradients in Gafchromic EBT film using a digital microscope," Med. Phys., 35, 3740-3747 (2008).

11) N. Nariyama, T. Ohigashi, K. Umetani, K. Shinohara, H. Tanaka, A. Maruhashi, G. Kashino, A. Kurihara, T. Kondoh, M. Fukumoto, K. Ono, "Spectromicroscopic film dosimetry for high-energy microbeam from synchrotron radiation," Appl. 
Radiat. Isot., 67, 155-159 (2009).

12) T. Tanaka, H. Kitamura, "SPECTRA: a synchrotron radiation calculation code," J. Synchrotron Radiat., 8, 1221-1228 (2001).

13) N. Nariyama, N. Kishi, S. Ohnishi, "Development of a portable free-air ionization chamber as an absolute intensity monitor for high-energy synchrotron radiation up to $150 \mathrm{keV}$," Nucl. Instr. Meth. Phys. Res., A524, 324-331 (2004).

14) H. Ohashi, K. Hirano (ed.), Synchrotron Radiation Beamline
Optics Technique Handbook, The Japanese Society for Synchrotron Radiation Research, ISBN 9784990054717 (1982), [in Japanese].

15) American Association of Physicists in Medicine, Radiochromic film dosimetry, AAPM Report No. 63 (1998).

16) F. Salvat, J. M. Fernández-Varea, J. Sempau, PENELOPE2008: A code system for Monte Carlo simulation of electron and photon transport, ISBN 978-92-64-99066-1 (2008). 